\title{
Chemical energy storage: Part of a systemic solution
}

\author{
ROBERT SCHLÖGL \\ Fritz-Haber-Institut der Max-Planck-Gesellschaft - Faradayweg 4-6, \\ D-14195 Berlin, Germany and \\ MPI für Chemische Energiekonversion - Stiftstrasse 34-36, \\ D-45470 Mülheim an der Ruhr, Germany
}

\begin{abstract}
Summary. - This paper is a primer into concepts and opportunities of chemical energy storage. Starting from the quest for decarbonisation we reveal the possibilities of chemical energy storage. We briefly discuss the critical role of catalysis as enabling technology. We concentrate on options of large-scale production of chemicals from $\mathrm{CO}_{2}$ and green hydrogen. We discuss one potential application of fueling future combustion engines that could run with minimal regulated emissions without exhaust purifications and legal tricks.
\end{abstract}

\section{1. - Introduction}

It is now clear that the world will act against climate change created by excessive combustion of fossil energy carriers. The world emits ca. $1 \times 10^{10} \mathrm{t}$ carbon atoms [1] per year from which ca. $75 \%$ stem from burning fossil energy carriers. Figure 1 shows a time series over recent history. Here the emitted carbon mass is given as $\mathrm{CO}_{2}$ : the conversion factor to the mass of carbon is 3.66 .

About half of the $75 \%$ come from power generation, the rest is shared between mobility and domestic/industrial heat production. Local and temporal variations are significant, creating a multitude of emission scenarios in the world. Here it is critical to see that replacing fossil power stations by solar electricity from wind, PV and other sources is not 


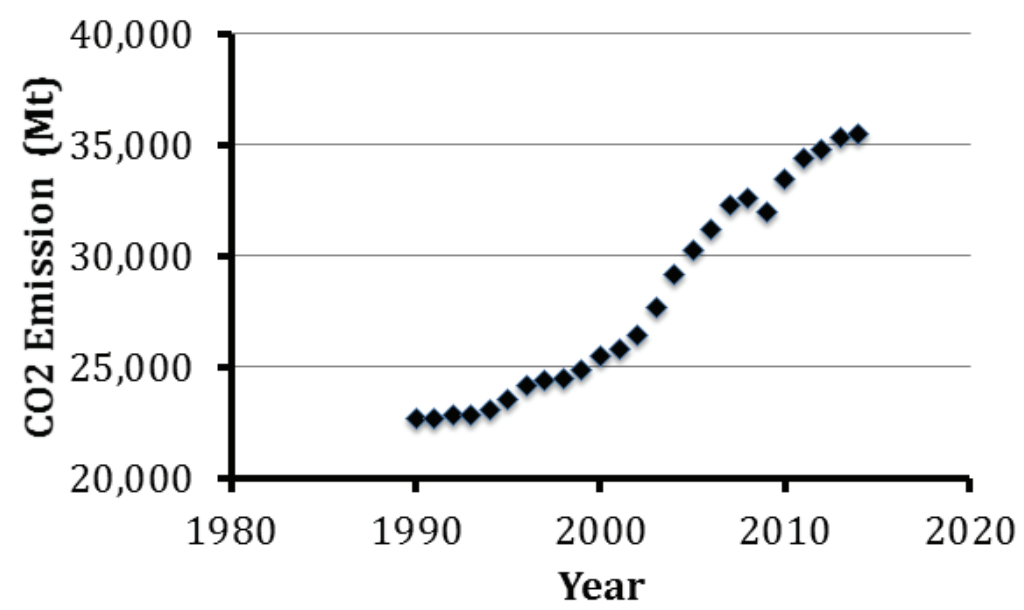

Fig. 1. - Temporal evolution of the global $\mathrm{CO}_{2}$ emission. All emission sources are collected in this graph.

sufficient to solve the climate change problem. Another key issue is the volatility of the green electricity being at variance to the demand profiles of power in many parts of the world. We may estimate that we can balance out without major energy storage about $50 \%$ of the load in a typical grid that is powered by green electricity [2]. The other $50 \%$ needs to be controlled power generation by combustion or nuclear energy or we need to overpower the solar generation capacity enormously and then we may end at about $75 \%$ green electricity. It is a key infrastructure to western societies (at least) to have electrical power uninterrupted whenever needed. The majority of the power goes into industrial applications where many processes run continuously $24 / 7$ and cannot easily adapt to significant a contribution of fluctuating energy supply.

If we demand that this property as well as unlimited mobility are conditional boundaries to sustainable energy systems then we have to accept that we need energy storage at world scale and not only locally for small-scale domestic applications in residential and rural areas. This is discussed [3] for example with combinations of PV and local battery systems. Such concepts are perfectly useful and understandable but create some largerscale problems. One is the societal dimension that people tend to forget the systemic nature of sustainable energy and think they have done their share. Economically such systems put the burden of energy system transformation on those who cannot build or afford such systems. Technically they sharpen the challenge for utility providers to hold capacities in place that are very rarely needed and thus maximal uneconomical. Useful would be smart systems of such architecture where the utility provider can control storage and supply according to the needs of the grid and not to the local needs.

This excursion highlights the complexity of measures in a systemic environment where locally "good" measures may have multiple detrimental effects in some other parts of the system. Such arguments also hold for the quest of decarbonisation through electrification 


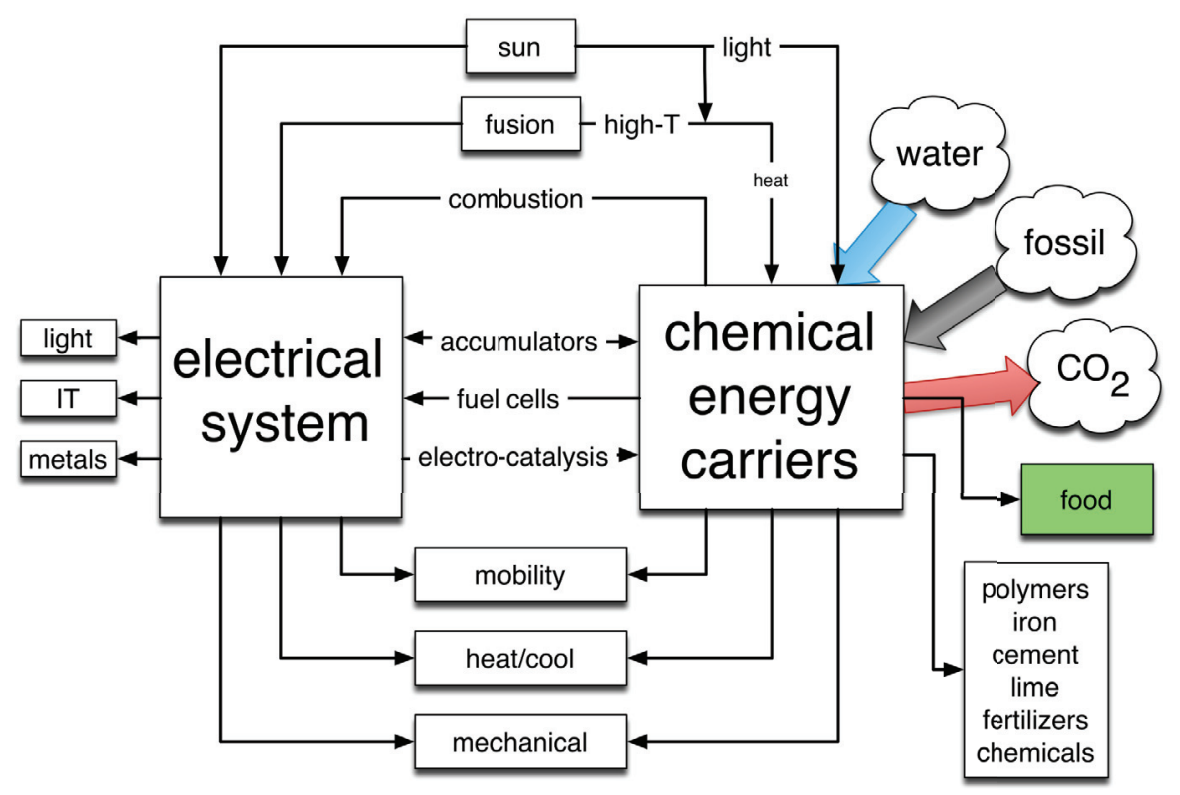

Fig. 2. - General structure of energy systems. Core applications are given. Either energy carrier type (large squares) can power some of them, some others are uniquely tied to the other carrier. Solar energy occurs both in form of photons and as heat resulting from interaction of solar photons with the atmosphere and hydrosphere of the planet.

of our society. Figure 2 shows that every energy system is always a combination of free electrons and chemical bonds as energy carriers. We are not free to omit one of them, as there are specific unique applications to each carrier type. We further see that we are good in converting chemical bonds into free electrons but that we cannot do the reverse very well. This creates the fundamental challenge of chemical energy conversion. We need to learn how to do conversion in both directions. This is independent of the not fruitful discussion about efficiencies; if we want to be sustainable then we have to minimize and in the long run abandon fossil energy carriers. True is that this process is not rapid, it will cover human generations and require multiple intermediate transformation paths. The size of the energy systems is simply too large for disruptive technical changes without disrupting the socio-economic conditions that are at least as critical for a successful transformation than technical and engineering challenges.

One reason why bi-directional conversion is so difficult is that converting chemical bonds into free electrons follows the laws of thermodynamics with fossil energy carriers. These laws state that chemical reactions are facile if they are downhill in free energy once we provide sufficient activation. Combustion is the perfect example for that, as $\mathrm{CO}_{2}$ and water are thermodynamically stable products. Back-conversion is more demanding as then we wish to store energy in chemical bonds, which by definition is energetically uphill and does not occur spontaneously. We see below how that is accomplished. 
Here we note that all fossil energy followed this uphill energy storage process that was enabled by solar energy and biomass in pre-historic times. Consequently, we should critically abstain to discuss "efficiency" of chemical energy storage in comparison to existing fossil energy carriers. This is often done to discredit sustainable energy systems in economic and technical terms being however a scientifically invalid argument.

One strategic element of chemical energy conversion is interfacial catalysis, regardless if it occurs in batteries, electrolyzes, photochemical devices or solar refineries. All chemical processes are subject to the dual delimiters of thermodynamics (uphill, downhill) and kinetics (slow, fast) that must not be intermixed. We cannot change thermodynamics but we can change kinetics by catalysis.

The science of catalysis [4] deals with the phenomenon that we can influence the rate of a chemical reaction by changing its course from a modus in which the reactants meet and either react or separate without reaction (homogeneous reaction) to a situation in which we use an auxiliary material (catalyst) that can be a molecule, a cluster, an enzyme, or a solid surface. This auxiliary material binds the reactants to it and allows for meeting of the reactants in their bound states. In many cases it acts not only in this "marriage broker" way but it also is a "bank" for redox equivalents (electrons) and donates to reactants electrons that the product has to give back to the bank in order to be released from the catalyst. The trick to controlling chemical reactions by catalysis is to separate meeting and reacting of the reactants in space and time and to "prepare" the reactants for their intended chemical transformation.

The challenge is to perform the reaction such that the catalyst is regenerated and can be used for more than one set of reactant transformation. In other words the binding between catalyst and reactant must be "intermediate" meaning strong enough to prepare the reactants but weak enough to allow their removal after reaction. The resulting "turnover number" per unit active site on a catalyst can be anything from a few hundreds to a few millions. We cannot well measure this important number as we have no clear picture about the active site. This is the structure of the catalyst that does the binding and exchange of reactants. For molecules as catalysts the number of options is naturally much less than for solids where a literally infinite number of configurations can exist. But as we cannot observe active sites at work and we do not know if they are continuously working or they take time off and stay in a "resting state" we have great problems to define the quantity "activity" in any catalyst.

Where we have fewer problems is to understand their energetic function. Let us assume that we want to store energy in $\mathrm{CO}_{2}$ by forming methanol as platform molecule using green hydrogen that we generate from water splitting. Figure 3 indicates a coarse profile through the potential energy landscape of this reaction. The details are known [5] but too complex to be used here.

The reaction is

$$
\mathrm{CO}_{2}+3 \mathrm{H}_{2} \longleftrightarrow \mathrm{CH}_{3} \mathrm{OH}+\mathrm{H}_{2} \mathrm{O}
$$

It is limited by thermodynamic equilibrium and does not run to completion under 


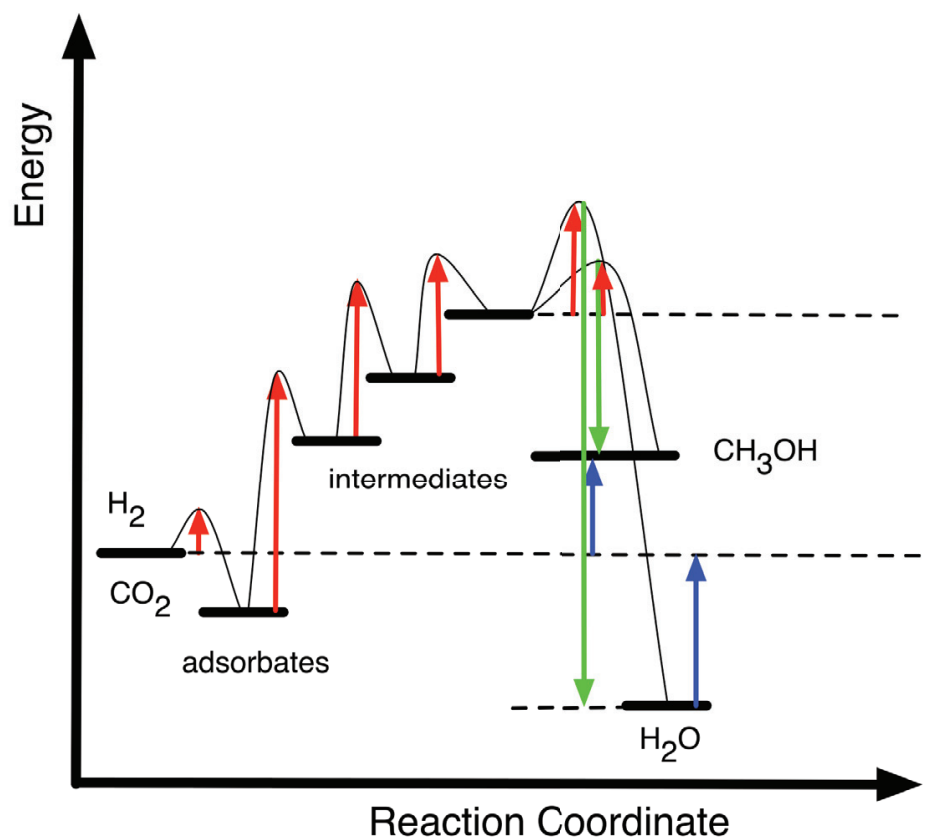

Fig. 3. - Schematic section through the potential energy landscape of the reduction of $\mathrm{CO}_{2}$ with hydrogen to methanol. We may use a modified copper catalyst, or Ru-organic molecules to perform the reaction. The red arrows denote kinetic energy barriers, the green arrows kinetic energy gain and the blue arrows thermodynamic energy quantities.

practical conditions. The reason is that we lack a catalyst that performs the reaction fast enough at low temperatures (around $400 \mathrm{~K}$ ). We need $600 \mathrm{~K}$ and a pressure of $60 \mathrm{bar}$ typically to obtain a technical relevant productivity. Another complication is that this reaction has a second product namely $\mathrm{CO}$ that is undesired. This further limits the conditions of operation of methanol synthesis.

From the diagram we see that we store energy in methanol, as it is higher in energy by about $0.5 \mathrm{eV}$ than the mix of $\mathrm{CO}_{2}$ and hydrogen. This is an uphill reaction that cannot occur for reasons of thermodynamics. The trick is to couple the reaction with a process that is downhill in energy and thus provides the driving force for methanol synthesis. This is the formation of water, which is not an unwanted byproduct but the reason for the ability to store energy.

The blue arrows in fig. 3 say nothing about the velocity of the reaction. The red arrows designate the activation energies from moving through the reaction coordinate (perform the making and breaking of chemical bonds) and say something about the reaction velocity Much of this barrier energy is re-gained upon completion of the energy (green arrows). The rest of it is energy loss, as we have to heat and pressurize the system to reach useful reaction velocities (about $1 \mathrm{~kg}$ of methanol per $\mathrm{kg}$ catalyst and hour). We see that we need to climb a high barrier of several intermediates before we reach at the climax an almost ready methanol molecule and a deactivated catalyst that 


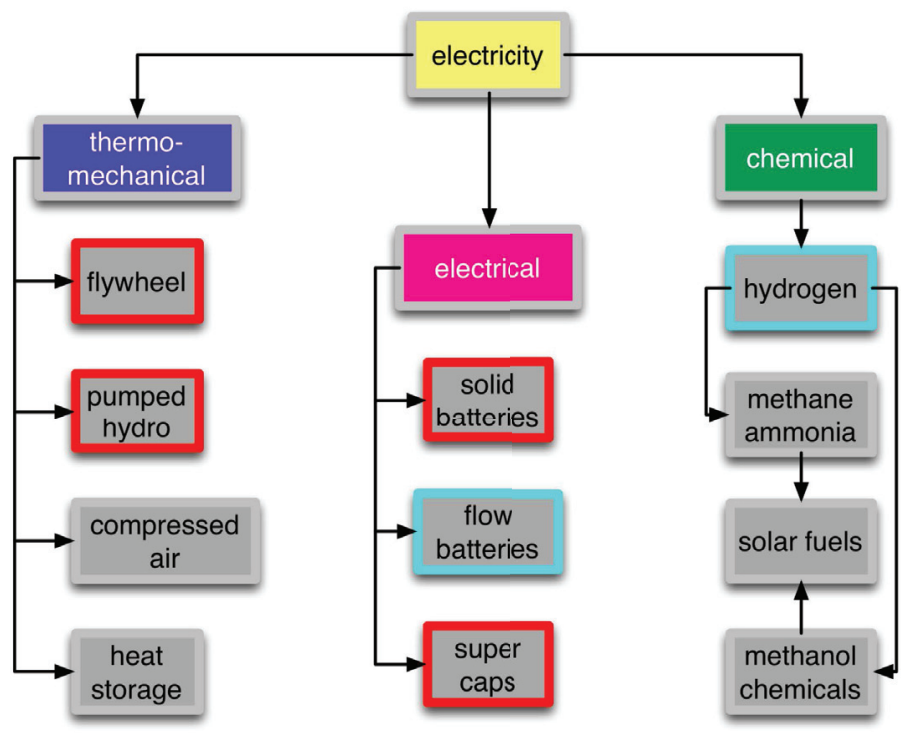

Fig. 4. - Energy storage options with physical and chemical means. The red boxes denote solutions that are used in present energy systems, the light blue ones are options almost ready for grid scale use, the other options are in demonstration or research status.

is charged with $\mathrm{OH}$ groups. It is the ingenuity of the chemist to minimize the number of intermediates and related activation barriers and to avoid that other reaction products occur during the journey over the higher energy barriers. When these barriers get very high it is unlikely that we obtain the wanted product and we produce unwanted products, such as carbon in the form of soot. This would be an expensive way of immobilizing $\mathrm{CO}_{2}$ that could be stored without danger in solid form, but as we will see, there are more effective ways of doing so.

If we now return to our energy issue we can ask what means of energy storage we have and if there are more convenient ways than chemical energy storage with all complexity standing behind the facts from fig. 3. In fig. 4 we give a systematic overview about storage options.

The multitude of options [6] becomes rapidly smaller when we consider their characteristics being storage capacity and storage times. We discriminate options for shaping the power system (very short time), for stabilizing the power system (hour timescale) and for long-term storage (seasonal time scales). All chemical energy storage solutions belong to the latter, as does pumped hydroelectricity. The other options are mostly suited for the other short-time purposes.

In fig. 5 we see a generic design of a sustainable energy system with some key components. Such a design takes care about the need of marginating both chemical bonds and free electrons as energy carriers. It is realistic in terms of utilizing solar energy as much as possible and allows for offering energy in the quality and distribution in space and time that we are used with fossil systems. 


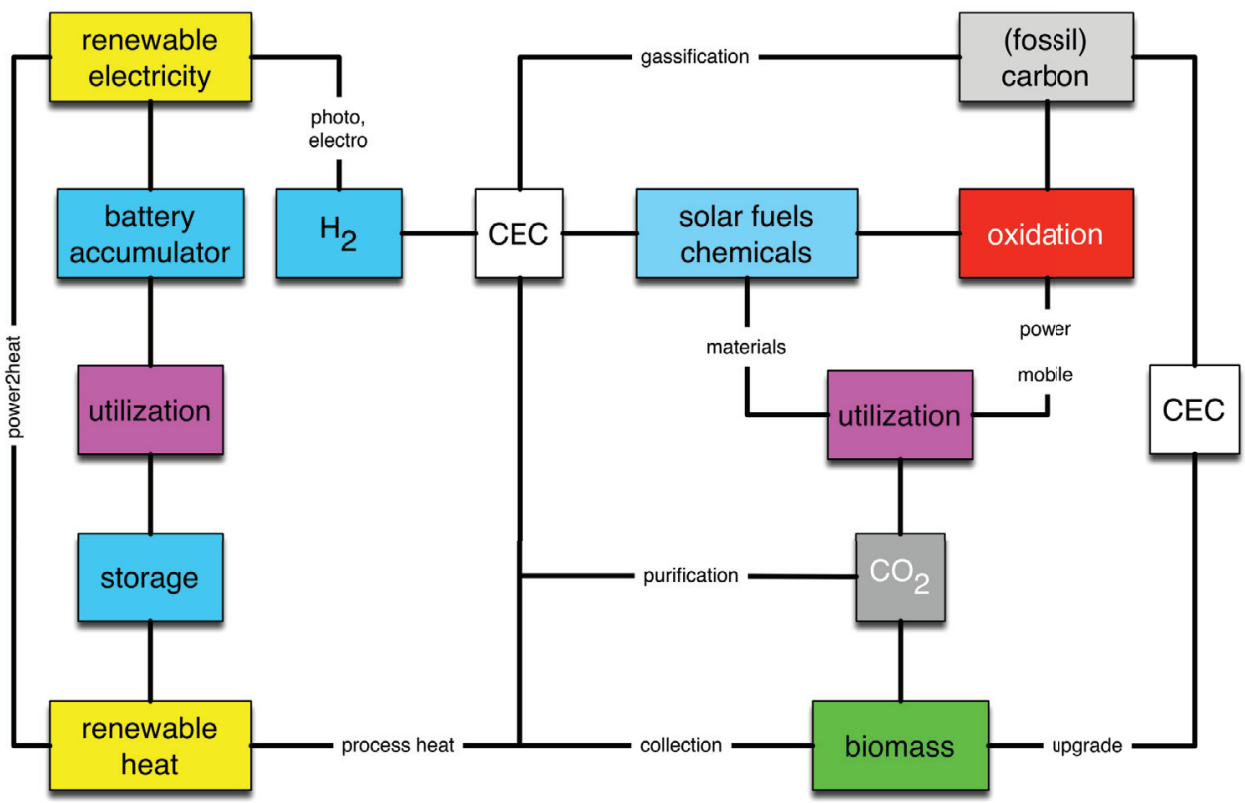

Fig. 5. - A generic design of a sustainable energy system. It uses primarily solar energy and converts it into free electrons and several forms of chemical bonds as energy carriers. Hydrogen is a critical intermediate but no consumer product. It uses as much electricity as possible and serves the needs of those elements in the energy system that cannot be electrified. It uses biomass as carbon collector and not as energy source. CEC stands for chemical energy conversion.

We will now concentrate on the long-term storage options as multiple information is available on battery science [7] being a critical part not only for grid stabilization but also for bringing green energy to the mobility sector. From fig. 5 we see that we can use green energy and green heat from the sun. We should use as much green electricity as possible immediately upon generation and provide a suitable large-area grid system with the necessary intelligent control system. Solar heat in low and high temperature form is easier to store than electrons for use as domestic heating (and cooling) application but also in some parts of the world as backup power station. All the rest of solar energy should be converted [8] in chemical bonds through the formation of hydrogen gas. Besides electrolysis [9] there is also plasma chemistry and combined photo-electrical conversion processes [10] at low and high temperatures that can be used. None of these concepts are ready yet for grid scale use. Also electrical water splitting is a difficult technology under the conditions of large-scale use [11] and variable electricity input; existing large systems run only on absolutely continuous electricity supply from hydroelectric installations.

Water splitting is a redox reaction in which oxide from water is oxidized to oxygen gas and the electrons gained from this are used to discharge protons into hydrogen gas:

$$
\begin{aligned}
2 \mathrm{H}_{2} \mathrm{O} & \longrightarrow \mathrm{O}_{2}+4 \mathrm{H}^{+}+4 \mathrm{e}^{-} \\
4 \mathrm{H}^{+}+4 \mathrm{e}^{-} & \longrightarrow 2 \mathrm{H}_{2} .
\end{aligned}
$$



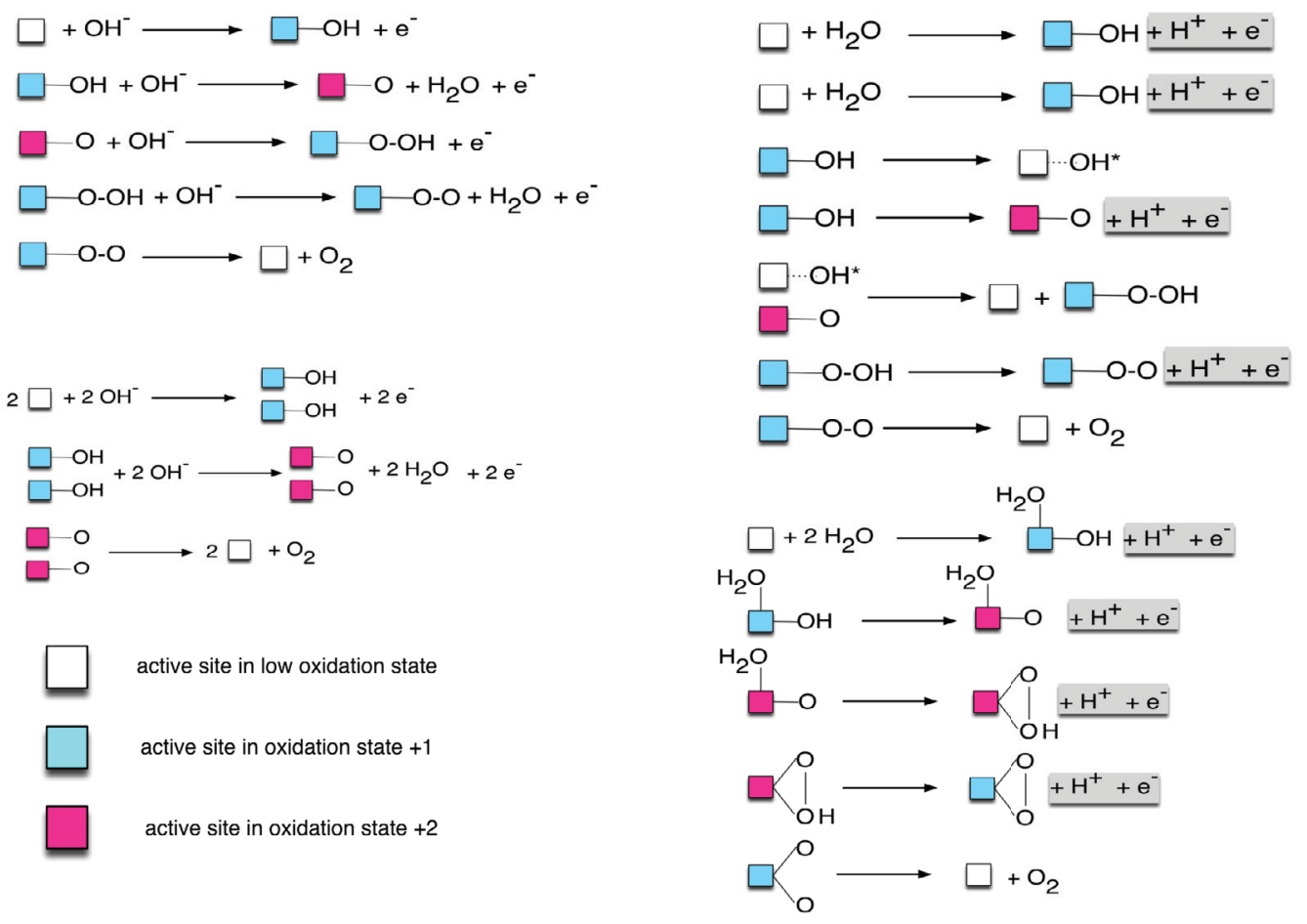

Fig. 6. - Reaction mechanisms of oxygen evolution in water splitting. The active site is denoted with colored squares, occurring in different oxidation states as indicated. The processes on the left side occur in basic media, the processes on the right side in acidic media.

The reaction is separated into two half reactions in electrical devices requiring two reaction spaces and two electrodes where the charges are separated from the ions. An advantage of this device is that the two reaction products hydrogen and oxygen are separated from each other which is highly advantageous. The disadvantage is that a membrane is necessary between the two compartments in order to allow the ions to migrate between the electrodes. The material science of these membranes and the limits they impose on reaction conditions are critical for the optimization of the device.

This reaction is carried out in nature as well as in electrolyzes or in photoelectrochemical cells. The exact nature [12] of the separation of charges and ions is different in these devices. We have learned much about the way in which nature does this reaction during photosynthesis [13] and we can use this knowledge for designing technical devices. We have to abstain from the idea to copy the biological process as it is way too complex for technical realization and it is also not efficient enough on the basis of hydrogen production per catalyst atom: the used enzymes are extremely complex and require continuous self-repair to remain operational.

We are far away from understanding on a robust basis the challenge of electrochemical water splitting with technical electrodes. We have a good idea about the possible 

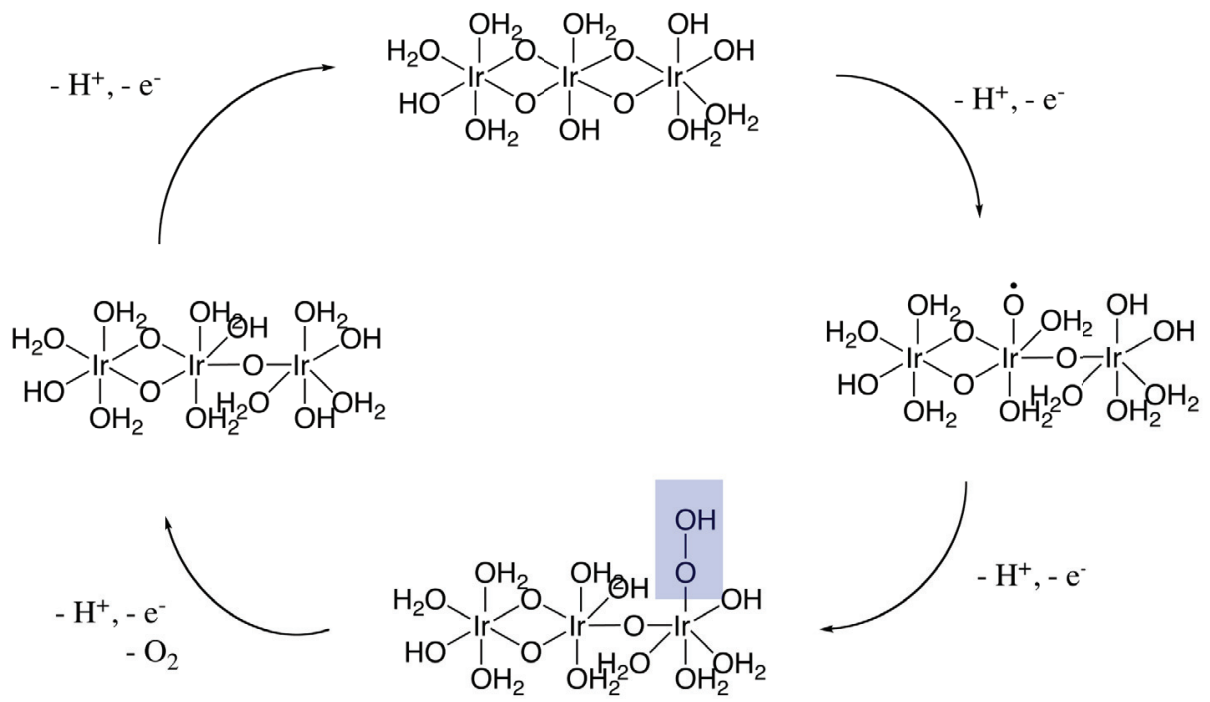

Fig. 7. - Likely reaction mechanism of oxygen evolution for water splitting. The critical intermediate is indicated.

mechanisms [14] and we know that not the hydrogen-forming part but the oxygen-forming part is the difficult reaction. We also have evidence that the reaction of oxygen formation is so difficult, as it requires an intermediate (an oxygen molecule with only one bond between the atoms) that is a very reactive species and that is likely to react with the electrode or hydrolyze with water rather than to finish the oxidation process and give off the final electrons.

In the last decades all likely mechanisms for oxidizing water in the so-called "oxygen evolution reaction" (OER) were formulated. It is surprising that such a simple reaction can be so complicated in detail. Figure 6 collects the likely reaction pathways. They are grouped according to basic or acidic environments. One can see that the choice of the condition of the water reactant plays a crucial role in the course of the reaction. Today one thinks that more than one reaction mechanism can co-exist in a working system. What is also clear from the figure is that the active site must be capable of changing its oxidation state, here assumed for two units per site. This is no problem for a metallic catalyst having the large number of electrons available at the Fermi level. For oxides that are required to withstand the aggressive character of the intermediates and allowing for facile adsorption of water this is a complicating requirement that can be fulfilled by several elements in the periodic table notably by the oxides of the late transition metals.

In fig. 7 we show a likely reaction cycle of oxygen evolution using the best known catalyst being $\mathrm{IrO}(\mathrm{OH})\left(\mathrm{H}_{2} \mathrm{O}\right)_{n}$. The critical reaction intermediate that can either form oxygen or form a peroxide species with the catalyst is shown. We note that the structure of the active species is quite flexible and does not resemble the notion of an inorganic rigid oxide. It can change the coordination of the metal atoms during reaction. It is believed that both properties may explain the excellent performance of the catalyst. 
Such hydrated species with other metal atoms tend not to be stable and condense into oxides under evolution of water. Such reactions are well known in the corrosion of iron to rust, for example. Many materials are being developed at present [15] with the hope to overcome the stability problems and poor performance of cheaper systems than Ir belonging to the rarest elements on the planet.

Let us assume we have solved the hydrogen problem and we have access to green hydrogen in the necessary amounts. Then we may form methanol from $\mathrm{CO}$ and hydrogen as platform molecule for many downstream products. In order to get a feeling for the quantities involved in such processes we discuss briefly the requirements of materials and the cost structure of such a process that does not yet exist in reality on grid scale. The world produces about $6 \times 10^{7} \mathrm{t} / \mathrm{a}$ methanol from $\mathrm{CO}_{2}$ and $\mathrm{CO}$ with hydrogen coming exclusively from fossil sources mainly from reforming methane with water. This process is cheap and easy to conduct in large installations and thus methanol plants can be very large factories with typically $1 \times 10^{6} \mathrm{t} / \mathrm{a}$ output. When we think about a methanol plant with green hydrogen the size of the electrolyzer plant may be controlling. Existing large electrolyzers have for example between 10 and $15 \mathrm{MW}$ power consumption that could come from a wind park. Then we see the following gross numbers for our plant. These are realistic numbers as not thermodynamic values but practically measured performances are used for the estimation. Nonetheless there will be additional losses for the production environment and the consumption of the plant utilities that are not taken into account. We obtain:

Electrolyzer $2100 \mathrm{Nm}^{3} / \mathrm{h}: 13 \mathrm{MW} / \mathrm{h}$ power.

Produces $950 \mathrm{kMol} \mathrm{H}_{2} / \mathrm{h}$.

Can reduce $315 \mathrm{kMol} / \mathrm{h} \mathrm{CO}_{2}$ to methanol.

Equals to $7.5 \mathrm{MMol} / \mathrm{d}$ or a usage of $330 \mathrm{tpd}$.

Equals to 120.000 tpa $\mathrm{CO}_{2}$ usage. (ca. $15 \mathrm{M} €$ p.a.)

This produces 88.000 tpa $\mathrm{MeOH}$. (ca. $32 \mathrm{M} €$ p.a.)

Power amounts to $115.000 \mathrm{MWh}$. (ca. $5 \mathrm{M} €$ p.a.)

Even when we consider that the cost of electricity assumed to be $4 \mathrm{ct} / \mathrm{kWh}$ may be too low, we recognize that the economics seems viable. There is, however, one critical condition. If many of such plants would operate, then the prize for methanol would fall drastically if no new utilization for methanol can be found. Here we see a general problem of chemical energy storage. The idea to replace existing chemical raw materials by green molecules finds quickly its end when we discuss chemical energy storage as a means to utilize $\mathrm{CO}_{2}$ (CCU in power2X processes) and not as a method to transfer green energy in other sectors of the energy system. The whole chemical industry in the world consumes about $4 \%$ of fossil resources and thus can only take up the same amount of carbon from $\mathrm{CO}_{2}$. On the other hand the generation of chemicals from fossil sources produces a lot of additional $\mathrm{CO}_{2}$ for using fossil sources (mainly gas) to provide the necessary heat and 
hydrogen through steam reforming. One way of using green hydrogen would thus be to power the chemical industry and to replace black hydrogen for hydrogenation of carbon and nitrogen (in the Haber-Bosch process for ammonia synthesis) by green hydrogen. This alone would reduce the carbon footprint more than the replacement of the fossil material sources (mainly naphta, a fraction of oil distillation).

So even if we can partly decarbonize chemical industry (this is still a formidable task to do) we need additional concepts to move green energy into the supply system. When we inspect figs. 3 and 5 we may realize that shortening the transformation chain of chemical energy conversion is a good idea to stay energy-efficient. Then we should discard the idea of $\mathrm{CO}_{2}$ hydrogenation and stick to hydrogen. The hydrogen economy [16] is a great idea and will likely play a role in upstream energy storage. The author is not convinced that this also holds for a end-user hydrogen economy as we have existing alternatives (see below) and do not want to bother end users with the unpleasant experience to deal with the infrastructure for the energy carrier pure hydrogen.

Using green electricity to generate hydrogen and back-convert this to electricity using a pipeline grid and (underground) storage is an option to counteract the volatility of green electricity. All technical elements of such a solution do exist except the water splitting devices compatible with stability under volatile load and with a cost structure acceptable for such an application. Such an option is, however likely more expensive that using cheap green electricity for heating applications where also $\mathrm{CO}_{2}$ can be saved and where no technological barriers exist. In later stages of the energy system transformation we will likely see not a competition but a co-existence of both options. Then we have a critical need to be able to split water for green hydrogen production.

What needs to be mentioned here is that the current debate about "excess green electricity" (that does not physically exist in a working power grid) is not the correct driver for creating a hydrogen generation technology of world scale. If we want to supply the whole energy system comprising power, mobility, heating and materials with green energy then we need much more primary green electricity than can ever result from the volatility excess of the power generation branch in the energy system. We need green electricity for the sake of doing chemical energy conversion and not consider this family of technologies as a remedy for excess electricity.

When we consider the priorities of useful applications of green hydrogen then we need to specify what is our priority of targets in changing the energy system. Avoiding $\mathrm{CO}_{2}$ emission as prime target clearly calls for re-generation of electricity, as all other options create $\mathrm{CO}_{2}$, and coal, the most economic source for electricity, the largest specific amounts of all energy carriers (ca. $0.8 \mathrm{~kg} \mathrm{CO}$ per $\mathrm{kWh}$ ). If we wish to replace $\mathrm{CO}_{2}$ most economically and generate the co-advantage of becoming less dependent on energy imports then we need to funnel the green hydrogen into mobility. This would reduce the dependence on oil being the largest volume of imported energy carriers in most countries. As oil carries substantial hydrogen with it (as octane $\mathrm{C}_{8} \mathrm{H}_{18}$ the combustion generates energy for 9 moles of water and 8 moles of $\mathrm{CO}_{2}$ ) its use generates specifically less $\mathrm{CO}_{2}$ than the use of coal in power generation. As we value mobility and thus fuel specifically higher than electricity the revenue of making artificial fuels is higher than re-generating electricity. 


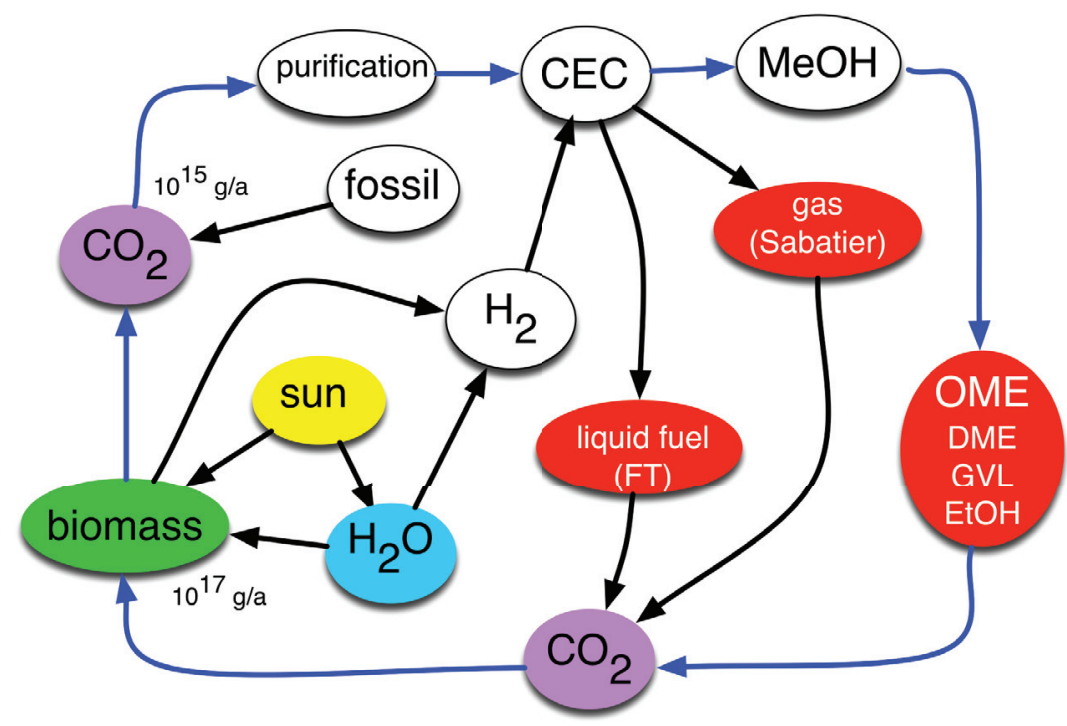

Fig. 8. - A closed carbon cycle for mobility. The blue arrows combine the elements forming the cycle. The red elements denote synthetic and designer fuels. The numbers specify the annual growth of biomass from which about $1 \%$ would be sufficient to collect all $\mathrm{CO}_{2}$ from present global mobility. The extent to which we use still fossil sources for other applications in the energy system defies the deviation from a fully closed cycle. This option indicates a transition path to arrive at a fully sustainable system.

When we have $\mathrm{CO}_{2}$ and hydrogen and want to create valuable liquid fuels then we have two choices. We can convert $\mathrm{CO}_{2}$ to $\mathrm{CO}$ and use the synthesis gas with the family of Fischer-Tropsch technologies for making artificial fuels replacing gasoline, jet fuel or diesel. This is practiced today at world scale using fossil gas sources to prepare synthesis gas and comes under the name GTL process (gas-to-liqid). The resulting fuels are of very high quality and free of impurities like sulfur and pay extra value at the filling station. This approach may be augmented by the addition of biomass to the reactant mix helping to minimize the atom economical waste of green hydrogen in forming the necessary but unwanted water molecules.

More innovative is to redesign the whole power train in vehicles in order to maximize the use of green electricity. We can combine an electric car with a mid-sized battery for short term driving and for recuperating deceleration energy with devices using on board green fuels. Some prefer green hydrogen and a fuel cell with the advantage of zero emission at the car and the disadvantage of having to install a new infrastructure (quasi worldwide). The author suggests to use liquid designer furls that can be distributed in the present fuel system but that have some advantages over present fuels without being incompatible with them. We may design molecules that burn without regulated emissions of $\mathrm{NO}_{x}$ and carbon particles. This indeed possible [17] when we use the insights from mechanistic studies [18] of combustion science. A co-condensation product of methanol with formaldehyde known as the family of oxymethylene Ethers (OME) is a perfect 
surrogate [19] for Diesel with the only disadvantage of having ca. half the energy density than Diesel. Similar solutions of molecules with C-O-C moieties rather than C-C-C groups can be found replacing gasoline. Only for jet fuel we have to resort to carbon-rich molecules for reasons of maximum energy density.

It we now complete our journey into chemical energy conversion then we can see how this technology can solve our energy problem without the quest for decarbonisation that is only partly possible as we have learned with fig. 2 . We should reduce the unbalanced use of fossil energy as much as possible. But we need not to stop using the great advantages of carbon-based energy carriers. All we need to do is to follow the example of nature and close the carbon flux cycle. When we combine the use of carbon energy carriers with the collection of $\mathrm{CO}_{2}$ and its re-reduction with green hydrogen then we can use without any harm carbon fuels in amounts limited by the availability of green hydrogen.

This is a far-reaching target but has the inherent advantage of not having to rely on reduction of energy consumption, which is adverse to the trends in global population and the socio-economic pressure of all humans having the equal right of energy access. If we wish to consider such architecture for sustainable energy systems [20] then we see that we are still at the very beginning of this transformation. We should concentrate to improve the bulk productivity of solar energy converters (PV, wind) as much as possible and not be satisfied with present technologies. All conversion technologies need enormous and disruptive changes in performance and cost structure for such a scenario. In fig. 8 we show such a scenario for the limited range of mobility in the sustainable energy system.

The simplicity of the scheme should not mask the enormous challenges for science and technologies as well as for economics and social comprehension and acceptance. This is a vision with little chance for realization in this generation. But without a master plan of priorities in science and technology we would not know how to evolve our capabilities in the energy challenge. As much as many fundamental discoveries from curiosity-driven science are needed as much also application-oriented science is required to select and evolve the science and understanding as basis for real technology evolution. At first we need some understanding about the scale and complexity of an endeavor "energy system transformation" that we seemingly stumble into without knowing much bout the inner structure of it. As little time as the climate change leaves us for the energy transformation, as detrimental would it be not to first structure the challenge and agreeing on a list of priority targets that will be different in different parts of the world. Global collaborations between scientists is the first and foremost requirement to ever meet the challenge and this in time for limiting the effects of climate change.

\section{REFERENCES}

[1] Aresta M. and Dibenedetto A., Dalton Trans., 2007 (2007) 2975, DOI:10.1039/B700658F; Riahi K., Kriegler E., Johnson N., Bertram C., den Elzen M., Eom J., Schaeffer M., Edmonds J., Isaac M., Krey V., Longden T., Luderer G., Mejean A., McCollum D. L., Mima S., Turton H., van Vuuren D. P., Wada K., Bosetti V., Capros P., Criqui P., Hamdi-Cherif M., Kainuma 
M. and Edenhofer O., Technol. Forecast. Soc. Change, 90 (2015) 8; Heimann M. and Reichstein M., Nature, 451 (2008) 289.

[2] Wagner F., Eur. Phys. J. Plus, 129 (2014) 20.

[3] Kaundinya D. P., Balachandra P. and Ravindranath N. H., Renew. Sustain. Energy Rev., 13 (2009) 2041.

[4] Schlögl R., Angew. Chem. Int. Ed., 54 (2015) 3465; Chorkendorff I. N. and J. W., Concepts of Modern Catalysis and Kinetics (Wiley-VCH, Weinheim) 2007.

[5] Studt F., Behrens M., Kunkes E. L., Thomas N., Zander S., Tarasov A., Schumann J., Frei E., Varley J. B., Abild-Pedersen F., Nørskov J. K. and Schlögl R., ChemCatChem, 7 (2015) 1105; Schumann J., Eichelbaum M., Lunkenbein T., Thomas N., Alvarez-Galvan M. C., Schlögl R. and Behrens M., ACS Catal., 5 (2015) 3260; Lunkenbein T., Schumann J., Behrens M., Schlögl R. and Willinger M. G., Angew. Chem. Int. Ed., 54 (2015) 4544.

[6] Gallo A. B., Simoes-Moreira J. R., Costa H. K. M., Santos M. M. and dos Santos E. M., Renew. Sustain. Energy Rev., 65 (2016) 800.

[7] Dunn B., Kamath H. and Tarascon J. M., Science, 334 (2011) 928; Shaahid S. M. and El-Amin I., Renew. Sustain. Energy Rev., 13 (2009) 625; Bruce P. G., Freunberger S. A., Hardwick L. J. and Tarascon J. M., Nat. Mater., 11 (2012) 19; TARAscon J. M. and ARmand M., Nature, 414 (2001) 359.

[8] Clarke R. E., Giddey S., Ciacchi F. T., Badwal S. P. S., Paul B. and Andrews J., Int. J. Hydrogen Energy, 34 (2009) 2531.

[9] Centi G., Quadrelli E. A. and Perathoner S., Energy Environ. Sci., 6 (2013) 1711.

[10] Yella A., Lee H.-W., Tsao H. N., Yi C., Chandiran A. K., Nazeeruddin M. K., Diau E. W.-G., Yeh C.-Y., Zakeeruddin S. M. and Graetzel M., Science, 334 (2011) 629.

[11] Gahleitner G., Int. J. Hydrogen Energy, 38 (2013) 2039.

[12] Rossmeisl J., Dimitrievski K., Siegbahn P. and Norskov J. K., J. Phys. Chem. C, 111 (2007) 18821.

[13] Ogata H., Kraemer T., Wang H., Schilter D., Pelmenschikov V., van Gastel M., Neese F., Rauchfuss T. B., Gee L. B., Scott A. D., Yoda Y., Tanaka Y., Lubitz W. and Cramer S. P., Nat. Commun., 6 (2015) 7890; Lubitz W., ReiJerse E. J. and Messinger J., Energy Environ. Sci., 1 (2008) 15.

[14] Viswanathan V., Hansen H. A., Rossmeisl J. and Norskov J. K., ACS Catal., 2 (2012) 1654; Rossmeisl J., Qu Z. W., Zhu H., Kroes G. J. and Norskov J. K., J. Electroanal. Chem., 607 (2007) 83.

[15] McCrory C. C. L., Jung S., Peters J. C. and Jaramillo T. F., J. Am. Chem. Soc., 135 (2013) 16977.

[16] Muradov N. Z. and Veziroglu T. N., Int. J. Hydrogen Energy, 33 (2008) 6804; Barreto L., Makihira A. and Riahi K., Int. J. Hydrogen Energy, 28 (2003) 267.

[17] Haertl M., Seidenspinner P., Jacob E. and Wachtmeister G., Fuel, 153 (2015) 328.

[18] Sarathy S. M., Osswald P., Hansen N. and Kohse-Hoinghaus K., Prog. Energy Combust. Sci., 44 (2014) 40.

[19] Iannuzzi S. E., Barro C., Boulouchos K. and Burger J., Fuel, 167 (2016) 49.

[20] Schlögl R., EPJ Web of Conferences, 98 (2015) 04001; Angew. Chem. Int. Ed., 54 (2015) 4436. 